\title{
Compliant Manipulation with a Dextrous Robot Hand
}

\author{
Paul Michelman \\ Peter Allen \\ Department of Computer Science \\ Columbia University \\ New York, New York 10027
}

\begin{abstract}
This paper addresses the problem of manipulating objects with a dexterous robot hand that are themselves in contact with the environment. A task-level formulation is presented and illustrated with several fundamental manipulation, translating and rotating a grasped object. Results of experiments are presented to demonstrate the feasibility of performing precision manipulations with a Utah-MIT hand.
\end{abstract}

\section{Introduction}

This paper ${ }^{1}$ discusses the control of precise, compliant manipulation tasks with multifingered robots. The focus of the work is performing manipulations of grasped objects that are themselves undergoing compliant motion. This important class of manipulations includes common tasks such as using tools, writing, and sliding an object on a surface. Our goal at Columbia's Center for Research in Intelligent Systems (CRIS) is the implementation of these tasks on actual systems. Therefore, we also present results of precision manipulation ${ }^{2}$ experiments performed with a Utah-MIT dextrous hand and discuss directions for future hand development.

The general manipulation problem has been defined as how to impart force and position trajectories on grasped objects in any position or orientation. We have chosen to address a subset of the general problem in which grasped object trajectories are applied only in specific, pre-defined directions. Several reasons for this choice are:

1. Task function itself often limits the number of degrees of freedom of a manipulated object. For example, a screwdriver can only apply force and move around its axis. Other motions are constrained by the screwdriver's contact with the screw slot.

${ }^{1}$ This work was supported in part by DARPA contract N00039-84-C 0165, NSF grants DMC-86-05065, DCI-86-08845, CCR-86-12709, IRI86-57151, IRI-88-1319, CDA-90-24735, North American Philips Laboratories, Siemens Corporation and Rockwell International.

2"Precision" is used in the technical sense that the motions of the grasped object are caused by finger motions rather than by arm or wrist motion (Napier [12]; Cutkosky [3])
2. Normally, a robot hand is attached to a robot arm. Gross positioning can be performed by an arm to align the hand with the environment. Therefore, only a limited number of predefined manipulatory motions need to be specified for a given robot hand.

The goal of this research, in which dextrous manipulation is driven by task function, is to develop a set of general skills which can be modified (i.e., parametrized) to deal with the variations from one task to another; for example, changes in geometry, friction coefficients, and masses and compliance of grasped objects. The basic manipulation strategy, however, is constant despite particular differences.

We suggest that a limited number of parameterized skills are required to perform a wide range of tasks. Datseris and Palm [4] maintain that dextrous hand should be able to manipulate a class of objects in three rotations and two translations. Elliott and Connolly [5], motor psychologists, developed a taxonomy of human manipulations that includes motions to rotate objects, translate objects, and multi-stage manipulations that allow regrasping during manipulation.

In this paper, we extend the previous functional paradigm to account for the necessary ability of a robot hand to cope with two levels of interaction forces: between the hand and the grasped object and between the grasped object and the environment. The capability to manage force interactions is required for several basic reasons:

1. Precise geometric models of a grasped object and the environment are often neither available nor desired. Force control allows tasks to be specified despite inaccurate models. While the exact positions of objects may not be constant from task to task, the amount of force required to achieve a goal may well be. Force control gives robots task flexibility and generalizability.

2. The progress-success or failure-of a task is partially assessed in terms of measured forces. In the screwdriver task, as pointed out above, the robot may have to measure the screwdriver axis torque to conclude whether to increase or decrease torque to turn the screwdriver in the desired way.

3. With particular regard to robot hands, the measurement 
and control of gripping forces allows a robot to adjust its grasp if there is imminent slippage. The grasped object is in contact with the environment, which exerts an indirect force on the grasped object and may cause the fingers to slip. By monitoring the gripping forces at each contact, the robot can increase the grasping forces before the onset of slip.

Each manipulation, then, has a high-level componentwhich specifies the gross position trajectories of each finger-and low-level components that account for maintaining grasp, generating force trajectories, and task monitoring and compensation. With few exceptions, previous research in dextrous manipulation has concentrated either on the high-level, task-level specification of manipulations or the low-level problems of grasp stability and grasp force specification. This paper discusses ways to unify the highand low-level components. Section 2 discusses related previous research in low-level (analytic) and high-level (empirical) manipulation. Section 3 enumerates the elements that are common to manipulation tasks. Sections 4 and 5 demonstrate the application of the paradigm for two precision manipulations. Finally, section 6 discusses the results of laboratory experiments.

\section{Previous research}

There have been a number of previous experimental demonstrations of elementary manipulations with robot hands. ${ }^{3}$ Okada [13] demonstrated turning a nut on a bolt and tilting a grasped object with a three-fingered hand. He derived finger trajectories and accounted for the evolution of the rolling contacts in some rotational motions. Fearing [6] twirled an object with a Stanford/JPL hand and analyzed the grasping forces during manipulation. Brock [2] has provided insights into the use of controlled slip during manipulation. Starr [16] demonstrated Cartesian compliance control for mating a grasped object with the environment.

As stated above, the empirical paradigm of Elliott and Connolly describes a set of precision manipulations that humans perform. Their descriptions are based only on the finger motions and not the force control strategies used, however. They neatly divide precision manipulations into three intuitive categories. (1) Simple synergies (used for translating a grasped object): The fingers are flexed simultaneously and convergently, as in a pinching motion; for example, scribing, squeezing. (2) Reciprocal synergies (used for rotating objects): The thumb adducts or abducts while the other fingers move relatively, e.g., a so-called "twiddle" movement such as tightening a small nut on a screw and removing the lid of a jar. (3) Sequential patterns: The fingers are moved repeatedly through a sequence of motions, while

\footnotetext{
${ }^{3}$ See Mishra and Silver $[10]$ for a summary of the basic issues.
}

perhaps regrasping the object in between motions, as when turning a screwdriver with one hand.

\section{A canonical set of precision manipulation tasks}

The set of possible skills is intimately related to the structural and sensing capabilities of the hand being used. ${ }^{4}$ Therefore, analysis of skilled tasks should not be considered separately from a target end-effector. A useful set of manipulations must be capable of performing most of the manipulations that a dextrous robot would be called upon to perform and incorporates a method for specifying both the functional and low-level aspects of precise manipulation. The method has two elementary parts: task partitioning, or what we call "functional dependency," and a workspace analysis, both of which are performed off-line and encoded into the task. Predefined, gross position trajectories are first formulated Then, during the execution of the task, the finger motions and forces are compensated using sensory feedback.

\subsection{Task partitioning}

Partitioning the fingers of a task according to function leads to a tractable way of describing manipulation. Several typical "roles" for the fingers have been identified: mover, comply (follower), fulcrum. As in hybrid control for a single robot, often the task specifications for the multiple fingers are orthogonal to each other. In section 4 we detail the orthogonal relationship of two fingers translating an object, while in section 5 the fingers take on the three roles mentioned above. For the moment, suffice it to say that the first goal in specifying a dextrous task is understand the functional dependencies of each of the fingers in the task: this use of high-level, heuristic knowledge simplifies the implementation of manipulation tasks and renders them tractable. Okada [13] analyzed two-fingered rotations of planar objects in which one finger is held stationary while the other imparts motion.

\subsection{Workspace analysis}

Once a task has been partitioned, many low-level mechanical details must be accounted for (called here "workspace analysis"). Here we list the important elements:

1. The kinematics of contact during manipulation (Kerr \& Roth [8]; Montana [11]): What type of contactsrolling or sliding-are used? What is the relationship between the motion of the contact point and the motion of the grasped object? After defining the object motion with respect to the fingertip, the joint angles that produce the desired contact motion are calculated.

\footnotetext{
${ }^{4}$ The human capacity to translate grasped objects by finger motions alone is quite limited. We seem to be able to translate objects roughly toward and away from the palm in a pinch-like move but not parallel to the palm. Still our hands are considered dexterous (by definition).
} 
2. Trajectory analysis: Although the precise finger trajectories are not known, gross-level position trajectories are included in the specification of the task. These are generally specified in the coordinate space of the manipulated object. In generating the trajectories of multiple fingers, it is necessary to make sure that chosen joint angles are within the workspace of each finger. This problem is more complex for multifingered hands than for single manipulators because solutions must be calculated simultaneously for a number of robots (Kerr and Roth [8]). The gross-level trajectories may be modified during the task using sensory data to account for modeling inaccuracies.

3. Strategies for sensing and controlling interaction forces during manipulation: Techniques for maintaining grasp stability and monitoring the progress of the task are critical. The forces are analyzed using elementary mechanics.

4. Geometric models of the grasped object and its interaction with the environment: What makes these tasks generalizable is their ability to work with a variety of geometries. For example, the pinch synergy described below works equally with a large block or a small cylinder. Although the robot need not know the precise task geometry a priori, a method of deducing the geometries from contacts is implicit in the encoding of the task.

\section{The Pinch Manipulation: Translating an object by pulling}

The first example is the following: use the pinch synergy (Elliott and Connolly [5]) to pull an object toward the palm of the hand using the thumb and opposing finger or fingers. For human hands, this is the fundamental technique for translating small objects perpendicularly to the palm. As a simple synergy, the joints of opposing fingers flex and extend simultaneously. In addition, there is an external translation force, $F_{e}$, which must be overcome. We can imagine pulling on small block that offers resistance. Both fingers begin in a extended position and gradually flex. As they flex, the block moves toward the palm. Figure 1 shows an example of two (two-dimensional) fingers pulling a block with the pinch synergy.

Task partitioning: A first look at the synergy might lead us to the conclusion that we could simply specify gross finger motions to perform the pinch synergy. Both fingertips would move toward the palm while applying sufficient friction to pull the block. For pure position control with stiff fingers, one requirement would be that both fingers not only follow the same trajectories, but do so at precisely the same rate. If they lose synchronicity, the block becomes unstable, rotating first one way then the other. To avoid this problem, the
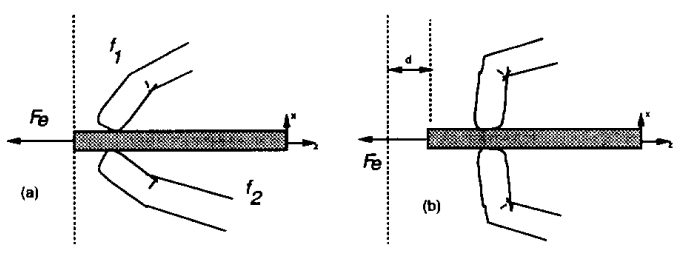

Figure 1: Pinch synergy

task can be partitioned in such a way that one finger exerts the pulling force while the other remains compliant in the pulling direction. The directions of compliance and stiffness are orthogonal to each other.

Figure 2 illustrates the task. Finger $1 f_{1}$ follows a position trajectory in the direction of motion. $f_{2}$, on the other hand, is compliant in the direction of motion, while remaining stiff in the direction orthogonal to motion. The friction force, $f_{2 z}$, pulls $f_{2}$, while $f_{2 x}$ keeps the block level. The task is described by orthogonalized force and position control directions as discussed in Raibert [15]. The $S$ matrices (where ' 1 ' represents position control) for $f_{1}$ and $f_{2}$ are:

$$
\mathbf{S}_{1}=\left[\begin{array}{lll}
0 & 0 & 0 \\
0 & 1 & 0 \\
0 & 0 & 1
\end{array}\right] ; \mathbf{S}_{2}=\left[\begin{array}{lll}
1 & 0 & 0 \\
0 & 1 & 0 \\
0 & 0 & 0
\end{array}\right]
$$

By using separate hybrid force/position controllers for each finger, the task can be naturally performed. The speed of motion and contact force are both controlled by $f_{1}$. The use of force control for $f_{1 x}$ permits the hand to control gripping force and also avoids the problem of inaccurate geometric modeling. The use of compliance in the direction of motion alleviates the problem of controller timing discrepancies.

Contact kinematics: During motion, the contact point moves along the fingertips. For a pure rolling contact, the distance travelled by the block is equal to the distance the contact point travels. The contact at time $t_{1}$ is located at $c_{c 1}$ and travels to $c_{c 2}$ during the manipulation. The Jacobian relating the contact point $c_{c i}$ to the finger frame $c_{f}$ is given by $J_{c f}, c_{f i}=J_{c f} c_{c i}$. Finally, given the position of the fingertip, the inverse kinematics yield the desired joint angles to generate the gross position trajectory. Using the task partitioning, this trajectory is the first approximation for $f_{1}$ 's motion. For the fingertips of the Utah-MIT hand, we assume an ellipsoidal shape for computing motions along the surface.

Grasp stability and task monitoring: The need to cope with interaction forces (such as changes in $F_{e}$ ) necessitates the sensing and control of the contact forces. If $F_{e}$ increases suddenly, the grasped object could slip. It is possible to monitor the contact forces to deduce the onset of slip before motion occurs. 


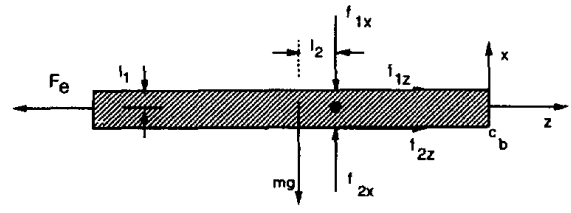

Figure 2: Freebody diagram of pinch synergy

The grasped object remains in equilibrium throughout the manipulation. Because of the low accelerations and relatively small masses of the fingers, we adhere to a quasistatic analysis (Fearing [6]; Trinkle [17]). The equilibrium conditions are well-known and are worked out (in two dimensions) with a few equations for the pinch task. From figure 2 ,

$$
\begin{aligned}
f_{1 x}-f_{2 x}+m g & =0 \\
f_{1 z}+f_{2 z}-F_{e} & =0 \\
l_{1} f_{1 z}-l_{1} f_{2 z}-l_{2} m g & =0 \\
f_{i z} & <\mu f_{i x}
\end{aligned}
$$

where $\mu$ is the coefficient of friction between the fingertip and the block. Therefore, the $f_{i z}$ are used during the manipulation to maintain stability and to assure that the $f_{i z}$ are sufficient to pull the rod in the presence of $F_{e}$.

The external force, $F_{e}$, can be calculated from equation 3. Increases in $F_{e}$ are reflected in an increased possibility of slippage; that is, $f_{i z} / f_{i x}$ approaches $\mu$, necessitating an increase in the normal force, $f_{i x}$.

Note that there is no unique solution to the choice of the initial grasping forces required so long as the equilibrium conditions are satisfied, since we have three equations (plus the no slip constraint) in four unknowns. A number of techniques for the allocation of initial contact force have been described (Salisbury [9]; Kerr and Roth [8]; Park and Starr [14]) in the general case. For the two-fingered pinch, it is required that the equilibrium conditions be satisfied: "sufficient force" to avoid slippage must be used.

This section has outlined the principle requirements of quasistatic manipulation for the pinch synergy. Notice that a robot must have at a minimum accurate force sensors to measure the contact forces, methods for measuring the contact location (contact sensors) and joint angles, and the ability to perform force and position control (or impedance control) in arbitrary Cartesian directions.

\section{The Twiddle Manipulation}

The twiddle manipulation, a reciprocal synergy, is an example of rotating a grasped object, similar to the twirling task accomplished by Fearing [6]. It is related to the common task of unscrewing the lid of a jar in that the thumb moves in a direction opposite to the opposing fingers. Figure 3 illustrates how it is accomplished with a three-fingered grasp. There is also a torque opposing the rotation which must be overcome. The details of the task - the contact kinematics and force control aspects-are derived in a way similar to the pinch synergy described above: we derive gross trajectories using the surface equations of the fingertips during rolling motion and the mechanics equations defining the equilibrium conditions.

One way of maintaining equilibrium equations for the twirling task is to give the three fingers equal weight during the manipulation. Although the internal grasping forces make this an underconstrained problem, constraints can be formulated to satisfactory the contact forces. An alternative is to partition the task functionally. In Figure $3, f_{2}$ is stationary throughout the task: it remains stiff throughout, serving as a fulcrum. The motion is imparted by $f_{1}$, while $f_{3}$ remains compliant. $f_{3}$ serves to stabilize the grasp and follow the motion of the object. The external force, $F_{e}$, provides a moment that resists the rotation. Using this partitioning, adjusting the torque applied to the block with the hand is accomplished by inreasing or decreasing $f_{1 y}$, the "mover's" normal force, leaving control of the net torque on the block to a single finger, rather than three.

The directions of control with respect to the finger change as the bar rotates. In practice, tactile sensors provide contact normal information can be used to monitor the control directions. Alternatively, positional contact sensors are also effective. The contact migrates around $f_{2}$ from $c_{1}$ to $c_{2}$. Assuming a circular cross section of the fingertips, the angle of rotation, $a$, is $\operatorname{arc}\left(c_{1}, c_{2}\right) / r$, where $r$ is the finger radius. ( $a$ is also found by noting the change in the angle formed by lines between contact points at $f_{1}$ and $f_{2}$ during the rotation.)

\section{Experimental results}

At the CRIS Robotics Lab, we are working with a Utah-MIT dextrous hand (Jacobsen [7]), a four-fingered, pneumatically controlled, tendon-driven manipulator. It is equipped with

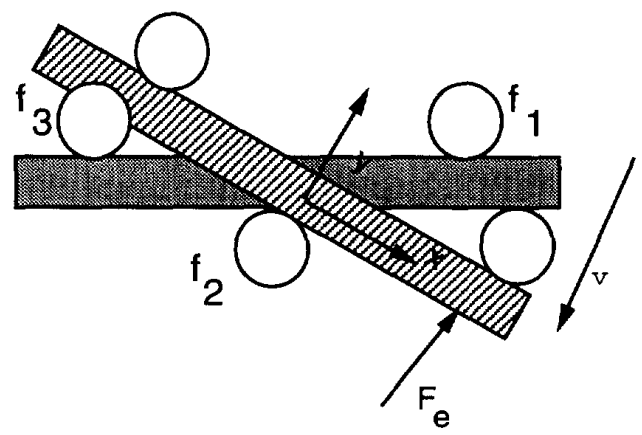

Figure 3: The twiddle manipulation 

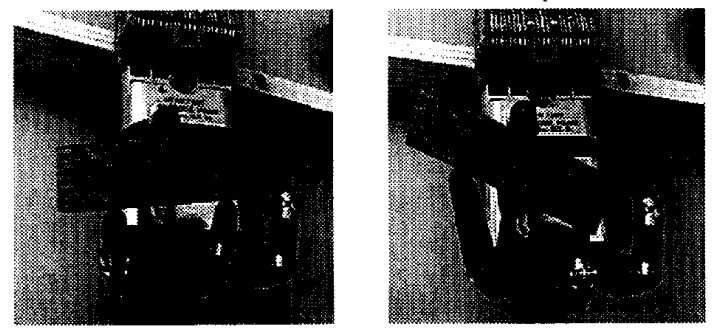

Figure 4: Utah-MIT hand rotating block: Clockwise rotation

Hall-effect joint angle sensors on each joint and tendon tension sensors to measure the tension on each of the 32 tendons. We have begun implementing the basic synergies described in this paper. Figure 4 shows the Utah-MIT hand rotating a block. The system is described in Allen, Michelman and Roberts [1].

Cartesian fingertip forces are approximated using the tendon tension sensors with the following relation: $F=$ $J^{-T} \tau=J^{-T}\left(T_{\text {extend }}-T_{\text {flex }}\right)$, where $\tau$ is the vector of joint torques for a finger, and $T_{\text {extend }}$ and $T_{f l e x}$ are the extensor and flexion tendon vectors for the joints of each finger. Problems of tendon sticking, hysteresis, sensor inaccuracies have made well-calibrated force measurement difficult. In addition, the values are unreliable near the Jacobian singularities. Nonetheless, the tendon tension sensors have proven to be monotonic at given finger positions, and the force measurements have been used qualitatively to assess increasing and decreasing contact forces reliably.

Compliance is achieved using a variant of stiffness control for each finger. Contact with an object causes a small displacement of a fingertip. Increasing the contact force results in an increased displacement. Assuming linearity, we know that $\mathbf{F}=\mathbf{K d x}$, where $\mathbf{K}$ is matrix of stiffnesses, and $\mathbf{d x}$ the displacements of the fingertips. Adjusting the displacement has the effect of increasing or decreasing the contact force, while keeping the displacement constant results in compliance with an external contact.

\subsection{Pinch manipulation and grasp reflex}

We performed the pinch manipulation. The hand uses three fingers-rather than two-in order to help stabilize the grasped object. Initially, the three fingers are spread wide enough to accommodate objects less than about $5 \mathrm{~cm}$ in width. Then, while the thumb is moved to grasp the object, the grasping forces are measured. When the thumb force in the normal $(x)$ direction exceeds a threshold, the grasping stops. Thereafter, the hand attempts to maintain the initial grasping force during manipulation. Next the hand is commanded to move the object down approximately $1 \mathrm{~cm}$ at a time, and to return the object to its initial position. Figure 4 (top) shows a typical initial grasp on a wooden block $(\approx 13 \times 6 \times 3 \mathrm{~cm})$. During the manipulation, the contact point migrates from the front surface of the fingertip, around the tip, to the back (fingernail) of the finger. Since these contacts approximate point contacts with friction at the fingertip, a two-fingered grasp proved unstable for the task. Several objects of different size and rigidity were similarly manipulated: a plastic box (audio cassette case, $\approx 1.3 \times 12.1 \times 7$ $\mathrm{cm})$, and a credit card.

To demonstrate the hand's ability to cope with $F_{e}$, the block was pulled by an external force to the point of slippage. The fingers successfully maintained the grasp in the presence of external disturbance forces. Our approach is to sense the relative increase of tangential $\left(f_{i z}\right)$ to normal force $\left(f_{i x}\right)$ during manipulation. As external forces bring about the slip of the grasped object, the normal force is increased to return the force angle within the cone of friction. The relative increase of the ratio $\left|f_{i z} / f_{i x}\right|$ indicates that the grasped object may slip. Naturally, different materials have different friction coefficients. When $\left|f_{i z} / f_{i x}\right|$ exceeds an empirically determined threshold, the $x$-component of the contact force is increased to lower the ratio, resulting in a stably grasped object. Figure 5 shows the resulting change in the ratio $\left|f_{i z} / f_{i x}\right|$. In this example, the threshold ratio $\left|f_{i z} / f_{i x}\right|$ was set to 0.35 . Initially, $F_{e}$ is zero. As the block is pulled in the $z$ (tangential) direction, the tangential force increases. Notice that it increases and then, after the $x$ component of the contact force is increased, returns to within acceptable boundaries. (Each tick in the time axis represents $300 \mathrm{~ms}$.)

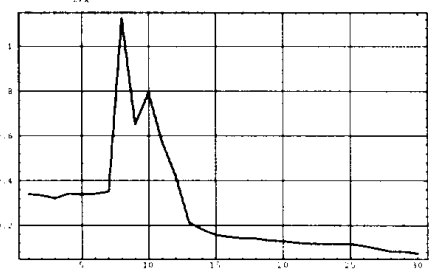

Figure 5: Slip Adjustment: Adjustment of $f_{i z} / f_{i x}$ to avoid slip

\subsection{Rotating a block}

The twiddle manipulation has been performed using the task partitioning described in Section 5. Referring to the functional dependencies, it was seen that one finger functioned as the mover and one as a fulcrum, while the third maintains compliance, stabilizing the grasped object. Here, the thumb was stationary while the first and second opposing fingers alternatively applied a manipulating force, pushing the block first one way then the other. For the initial experiments, the inherent joint compliance of the Utah-MIT hand was used. The compliant finger tracked the mover finger in the opposite direction, while maintaining contact with the grasped object. Initially, objects ranging in thickness from $1 \mathrm{~mm}$ (a 
credit card) to over $2.5 \mathrm{~cm}$ (a wood block) were successfully and repeatedly rotated 60 degrees back and forth. (Figure 4 shows a rotation of about 30 degrees.) To attain the initial grasp, the grasping force on the fulcrum is monitored until it is within an empirically acceptable range.

In addition, the external force applied to the block and motion caused by the external force have been measured. While the block is held, an external torque is applied to the block. The change in finger forces is used to determine whether the torque is clock wise or counterclockwise, and the relative magnitude of the torque, increasing or decreasing.

\section{Discussion and future work}

Experiments to date show that basic manipulations involving measuring and controlling forces applied to grasped objects are possible with a Utah-MIT hand. Our initial work utilized the robot's passive joint compliance, rather than actively controlling joint stiffness, as suggested in section 3 . Task partitioning, we believe, will simplify the control of external forces and grasped object trajectories. In addition, tactile sensors, capable of measuring contact positions with 1-2mm will shortly be added to the fingertips to eliminate errors in the estimate of contact normals and control directions.

Our work thus far has concentrated on building the components of a system that can perform precision, compliant manipulations with a dextrous hand. Thus far, we have demonstrated the ability to perform elementary rotations and translations, monitor interaction forces between the grasped object and the environment, and compensate for slippage before it occurs. We work toward making the system more robust and autonomous and building more complex synergies, in particular, the reciprocal synergies, which require the regrasping of objects during manipulation, as in using a screwdriver with one hand.

\section{References}

[1] P. Allen, P. Michelman, and K. Roberts. A system for programming and controlling a multi-sensor robotic hand. IEEE Transactions on Systems, Man, and $C y$ bernetics, 20(6):1450-1456, 1990.

[2] D. L. Brock. Enhancing the dexterity of a robot hand using controlled slip. In 1988 IEEE International Conference on Robotics \& Automation, pages 249-251, 1988.

[3] M. R. Cutkosky. On grasp choice, grasp models, and the design of hands for manufacturing tasks. IEEE Transactions on Robotics and Automation, 5(3):269279, June 1989.

[4] P. Datseris and W. Palm. Principles on the development of mechanical hands which can manipulate objects by means of active control. Transactions of ASME Journal of Mechanisms, Transmissions and Automation in Design, 107:148-156, June 1985.
[5] J. Elliot and K. Connolly. A classification of manipulative hand movements. Developmental Medicine and Child Neurology, 26:283-296, 1984.

[6] R. Fearing. Implementing a force strategy for object re-orientation. In 1986 IEEE International Conference on Robotics \& Automation, pages 96-102, 1986.

[7] S. Jacobsen, E. Iversen, D. Knutti, R. Johnson, and K. Biggers. Design of the Utah/MIT dextrous hand. In 1986 IEEE International Conference on Robotics \& Automation, pages 1520-1532, 1986.

[8] J. Kerr and B. Roth. Analysis of multifingered hands. International Journal of Robotics Research, 4(4):317, Winter 1986.

[9] M. T. Mason and J. K. Salisbury. Robot hands and the mechanics of manipulation. MIT Press, 1985.

[10] B. Mishra and N. Silver. Some discussion of static gripping and its stability. IEEE Transactions on Systems, Man and Cybernetics, Vol 19(4):783-796,July/August 1989.

[11] D. Montana. Tactile sensing and the kinematics of contact. PhD thesis, Division of Applied Sciences, Harvard University, 1986.

[12] J. Napier. The prehensile movements of the human hand. J. Bone Joint Surg., 38B(4):902-913, 1956.

[13] T. Okada. Computer control of multijointed finger system for precise object-handling. IEEE Transactions on Systems, Man, and Cybernetics, SMC-12(3), May/June 1982.

[14] Y. C. Park and G. P. Starr. Finger force computation for manipulation of an object by a multifingered robot hand. In 1989 IEEE International Conference on Robotics \& Automation, pages 930-935, 1989.

[15] M. Raibert and J. Craig. Hybrid position/force control of manipulators. ASME Journal of Dynamic Systems, Measurement, and Control, 102:126-133, June 1981.

[16] G. P. Starr. Experiments in assembly using a dexterous hand. IEEE Transactions on Robotics and Automation, 6(3):342-347, June 1990.

[17] J. C. Trinkle. A quasi-static analysis of dexterous manipulation with rolling and sliding contacts. In 1989 IEEE International Conference on Robotics \& Automation, pages 788-793, 1989. 